\title{
71. OLD AND INTERMEDIATE POPULATIONS IN THE MAGELLANIC CLOUDS
}

\author{
W. G. TiffrT
}

Lowell Observatory

It is becoming increasingly evident that the older stellar components of the Magellanic Clouds are significantly younger, on the average, than the old star component of the Galaxy. Magellanic Cloud red globular-like clusters of the NGC 419 variety, and the common field stars of similar type, are almost certainly to be classified as intermediate-age populations by galactic standards. The oldest galactic population, seen in the galactic halo, globular clusters, and oldest galactic clusters, is apparently very sparse in the Magellanic Clouds, although in the SMC at least something very similar is seen in and near NGC 121.

It seems probable that the differences in stellar population between the Clouds and the Galaxy can be explained in terms of differing dynamical conditions for star formation such that the relative numbers of stars formed in various stages of the history of the systems have been quite dissimilar. In particular, it appears that during the time of very active initial star formation in the Galaxy stars were formed in only small numbers in the Clouds. Later when galactic star formation had declined, slowly accelerating formation rates in the Clouds finally began to produce the myriads of common "older" stars we see today. This slowly developing wave of star formation has probably continued more or less smoothly up to the present day.

Although we cannot expect to find the relative numbers of different ages and types of stars to be the same in the Galaxy and the Clouds owing to dynamical differences, we can reasonably expect the same types of individual stars. This is the case since stellar models are rather specific once the star formation process has been set in motion. Even here, however, one should not expect perfect identity since chemical enrichment or "chemical evolution" is dependent upon the number and masses of stars formed. Hence dynamical evolution controls the rate at which material is chemically enriched. In systems with differing dynamical evolutionary patterns stars of a given type and age can therefore form out of media with slightly different chemical composition. Depending upon the sensitivity of stellar models to small chemical changes the stellar types themselves may therefore differ in various details between galaxies. This point has particular bearing on the intercomparison between cepheid variables in the Galaxy and in the Clouds. There is some evidence that Cloud cepheids are somewhat more luminous than cepheids of the same period in the Galaxy. This observation is based upon a comparison between the cepheids and cluster-type variables by utilizing their galactic calibrations. It tentatively seems that either the Cloud cepheids are brighter, or the Cloud cluster-type variables are fainter, than in the Galaxy. For reasons to be discussed further later, the older cluster-type variables probably provide the more reliable comparison. It is worth emphasizing at this point the very great value of the Clouds as objects within which very accurate differential comparisons can be made between classes of stars. Such 
comparisons are independent of galactic calibrations and can both guide the galactic calibration and point the way toward testing the constancy of our calibrations under varied chemical and dynamical evolution patterns.

Under the reasonable assumption of initially similar composition in the prestar media of the Galaxy and Clouds we can expect to find truly identical types of stars (although in very different numbers) among the very oldest stars. If the initial media are not similar there is even less basis for comparison of young and intermediate age stars between galaxies since these stars are chemically affected by the differing evolution patterns as well as by initial composition. Clearly, without

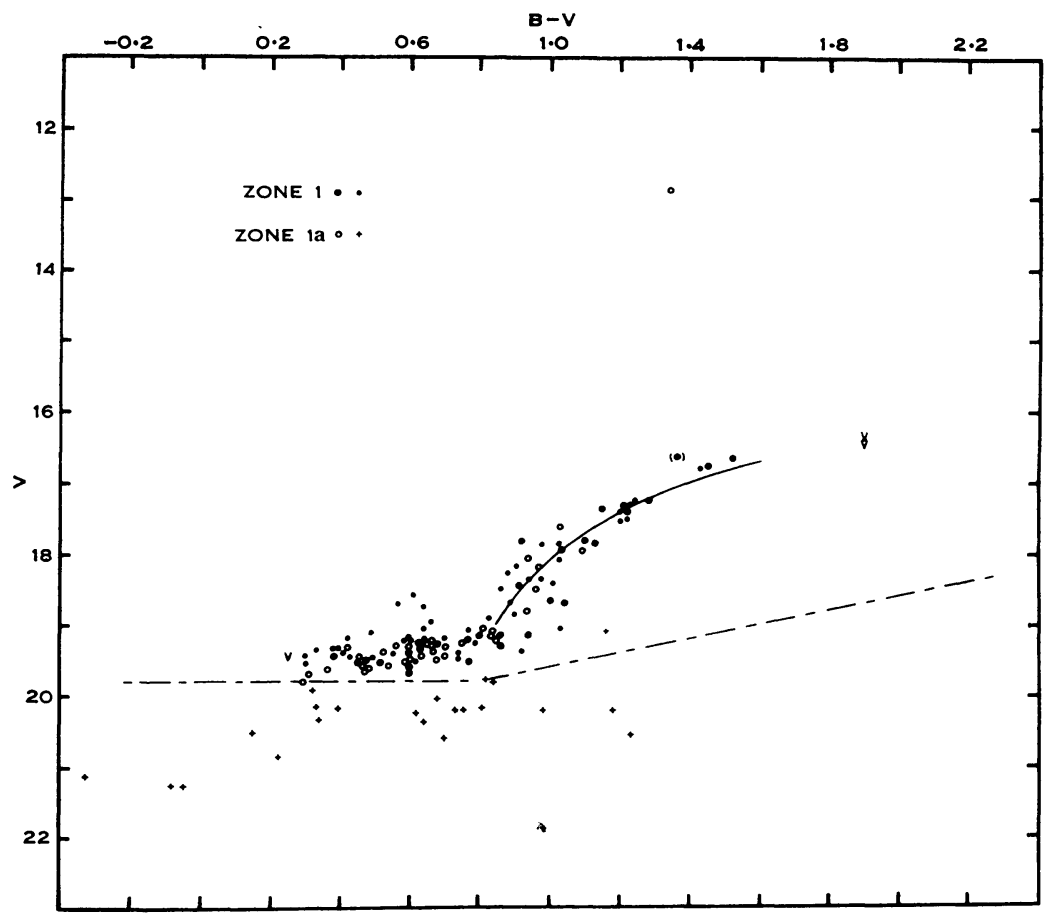

Fig. 1.-The innermost regions of NGC 121 which could be photometered without serious crowding. Zone $1 \mathrm{la}$ is more reliable than zone 1 and within zones the quality is indicated by different symbols. Variable stars are indicated with a V.

detailed information on the evolutionary processes, the oldest stars provide the most reliable comparisons between galaxies. Starting from the oldest stellar types and progressing to younger and younger types of stars, we can theoretically follow the separate patterns of evolution in the Galaxy and the Magellanic Clouds.

There is no difficulty in finding examples of the intermediate age stellar component in the Magellanic Clouds. Most of the red globular-like clusters and associated fields apparently fall in this category. There will probably be no fundamental difficulty in gradually ordering these clusters in an age sequence with the young blue globular-like clusters in the Clouds. NGC 419, which may be taken as typical 
of a large part of the Magellanic Cloud intermediate age component, contains a giant branch which is both more luminous and redder in extreme values than the old globular clusters in the Galaxy. The giant branch may also be split into two parts. The presence or absence of a horizontal branch in such clusters has not been conclusively shown, although cluster type variables are fairly certainly not present.

At the present time our knowledge of the oldest stellar component in the Magellanic Clouds is largely limited to the NGC 121 region of the SMC. In Figure 1 the colour-magnitude diagram of NGC 121 itself is shown. The precision of the photometry in the crowded areas near the cluster is not especially high, but the general features of the diagram are clear. A smoothly continuous giant branch terminates near $B-V=\mathbf{1} \cdot \mathbf{6}$, except for two red variables, and a strong horizontal branch including cluster-type variables is present. The vertical subgiant region

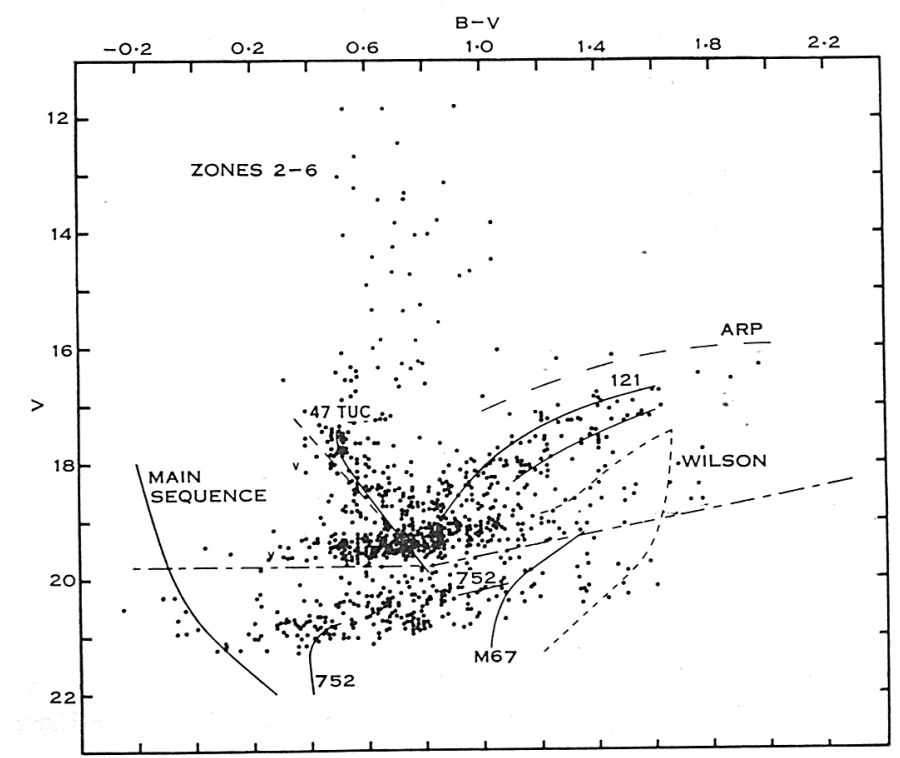

Fig. 2.- The outer regions of NGC 121 and the SMC halo. Much of the foreground is contributed by 47 Tuc.

has a colour index $B-V=0 \cdot 8$. In all these characteristics NGC 121 resembles reasonably well the old galactic globular clusters, and it is on the basis of this comparison that NGC 121 is considered to be quite old. It should be noted that the NGC 121 giant branch is significantly fainter than giant branches in NGC 419 type intermediate age clusters.

NGC 121 lies sufficiently far from the SMC core for the region to be free of young stars; the area around the cluster is therefore ideal for investigation of the old SMC halo. Figure 2 shows the colour-magnitude diagram for this area. The brightest stars, and all the stars above the horizontal branch, belong to the galactic foreground, especially to 47 Tucanae, and will not be considered further here. The red giant region contains NGC 121 type giants and fainter giants, but no significant number of NGC 419 type giants which would fall along the dashed line in the figure. 
Faint red stars, apparently the SMC equivalent of old and intermediate age galactic Population I giants, are apparently present in the halo. Thus we have the side by side occurrence of old giants of both classical populations. The faint red giants may offer a good opportunity to study the oldest SMC stars. Although difficult to study owing to faintness, the outer envelope of the region occupied by these stars could provide an upper age limit for the SMC. The rarity of really old globular clusters may make this the best means for determining an age limit. It seems clear already that the SMC halo contains some stars nearly as old as the oldest in the Galaxy. The youngest stars in the NGC 121 region would seem to be about $1 \times 10^{9}$ years old based upon the tentative identification of faint bluish stars as $\mathbf{F}$ subgiants.

The wide luminosity spread among the giants in the NGC 121 region probably implies a wide dispersion in metal abundance. This luminosity dispersion is not obvious on the horizontal branch, however, and horizontal branch luminosity would not seem to be particularly sensitive to metal abundance variation. A possible

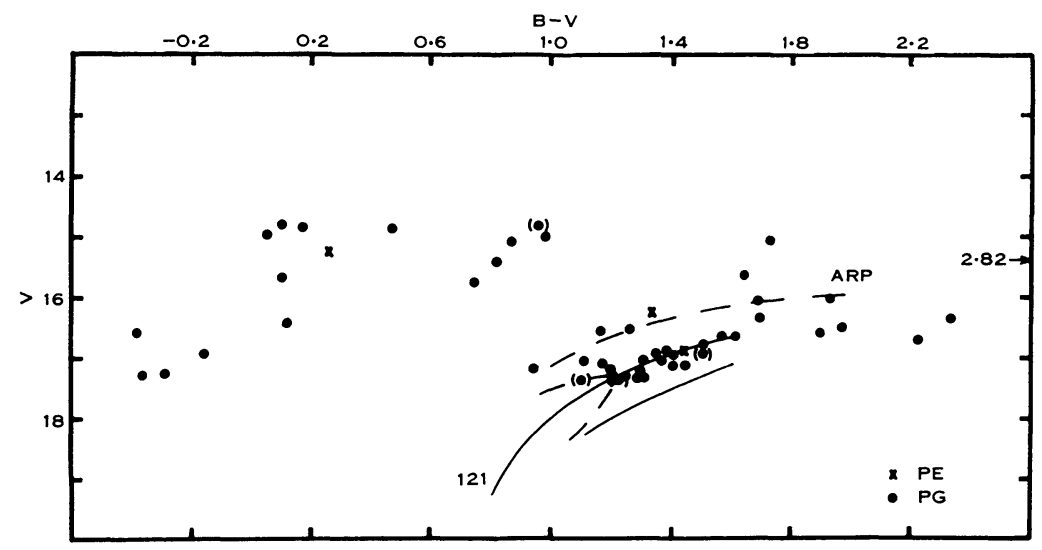

Fig. 3.-A preliminary sample of stars in the inner SMC halo near Kron 121 .

dependence of horizontal branch luminosity on age is suggested in the sense that the younger stars are the most luminous. This effect is supported by the relative faintness of the NGC 121 horizontal branch and general mass-luminosity expectations. The NGC 121 region cluster-type variables lie at $\langle V\rangle=19 \cdot 6$; there does not appear to be any appreciable reddening in the region.

Although NGC 121 and the surrounding field can be favourably compared with stellar components of the Galaxy there remain significant small differences. NGC 121 combines a horizontal branch concentrated toward the red end with giants which lie nearly 3 mag above the horizontal branch in luminosity. In the Galaxy the red concentrated horizontal branches are associated with giants only about $2 \cdot 3$ mag brighter; 47 Tucanae is an example. Furthermore, there seems to be a distinct gap in the subgiant extension of the giant branch just below the horizontal branch. Such a gap is not present, or at least is not as strong, in clusters in the Galaxy. Finally, one might mention the curious flattening observed in many Cloud clusters, 
including NGC 121, suggestive of relatively high angular momenta. These differences are small compared with the differences encountered when comparing NGC 419 with the Galaxy, however, and the interpretation in terms of age seems clear.

In passing from NGC 121 toward the SMC core one would expect and indeed does encounter progressively younger stars mixed with the old. Figure 3 illustrates a preliminary result near Kron 21, a sparse apparently intermediate age cluster. NGC 121 type old giants are seen in the region but stars equivalent to NGC 419 giants also appear along with some fairly young blue stars. No extremely young stars are seen in this inner halo region. By extending studies to additional regions we may hope sometime to build up a logical pattern of ages and from this derive information about the chemical and dynamical development of the SMC.

The situation is much less clear in the Large Magellanic Cloud at this time. Intermediate age systems are obviously present and some have been studied in detail. However, no older equivalent of NGC 121 has been certainly identified. A preliminary study near NGC 1850 in the west end of the LMC bar suggests that the bar contains red giants of the NGC 121 variety; this remains to be confirmed, however.

Investigation of the old and intermediate age stellar population in the Clouds has barely begun. Certain differences between the Galaxy and Cloud stellar populations certainly appear to be present and must reflect differences in the dynamical and chemical evolutionary patterns of the galaxies. These differences should provide means of studying the evolutionary patterns. At the present time we should neither over-emphasize nor underplay differences or similarities between the Clouds and the Galaxy. Rather we should move toward the yet future time when we have enough data on many different types of objects and their interrelationships to provide a broad base for more definitive studies.

\section{Discussion}

Westerlund: I have suggested that most yellow and red stars in the NGC 602 region are foreground stars. However, I admit that the magnitude scale used by van Rhijn is somewhat uncertain, and it appears possible that about 20-50 of these stars are in the SMC. It is reasonable to expect a few members of the SMC halo in this part, as I have found that the globular cluster L113 is very similar to NGC 121. I agree that it is certainly worth while searching for RR Lyrae variables in this region and around NGC 602.

Bok: In the interpretation of these results one should be very careful not to stress too much the colour features that depend closely on stars near the plate limits in $B$ and in $V$.

Tifft: Most of the significant features I have considered in the NGC 121 region are well above the plate limit. In the SMC wing region where Westerlund has carried out a survey (NGC 602) the NGC 121-like horizontal branch feature is near the limit of photometry, but the concentration is very distinct and agrees so closely with the NGC 121 horizontal branch that I do not believe one can seriously doubt the identification. One may doubt the giant region somewhat, but even there the presence of the horizontal branch implies that giants can and in fact must be present. Even near the plate limit if data are carefully combined so as to minimize random errors, one can detect clumping of stars if they exist. Calibrations may be systematically in error near the plate limit, but this does not prevent detection of specific groups of photometrically distinct objects - we simply make systematic errors in locating the groups on our diagrams. With all these factors in mind I believe we can work very close to plate limits but one must obviously use reasonable caution in interpreting results from such photometry.

Woolley: The words have been taken out of my mouth by Professor Bok, but I would like to emphasize the extreme danger of theorizing about concentrations of stars near the plate limit. 
Arp: The superposition of colour-magnitude diagrams from my field regions over the SMC agrees excellently with Westerlund's diagram of field stars in the SMC. I would like to point out that it was shown that the superposition of colour-magnitude diagrams for individual clusters in the SMC gives a very similar diagram. All the broad features can be recognized in these composite field colour-magnitude diagrams. The sprinkling of galactic foreground stars over these small area regions of the SMC should not interfere seriously with the interpretation of the diagram.

Eggen: There may be a spread in ages.

\title{
72. THE RED GLOBULAR-LIKE CLUSTERS IN THE MAGELLANIC CLOUDS
}

\author{
S. C. B. Gascoigne
}

Mount Stromlo Observatory

There are about 50 clusters in the Magellanic Clouds which from their spherical symmetry, integrated colours, and luminosities appear similar to the globular clusters in the Galaxy. The colour-magnitude diagrams of these clusters should give moduli for the Clouds, indications of the age and chemical composition of the clusters themselves, and perhaps some information about the evolutionary tracks of old stars generally. The first investigation of this kind was carried out by Arp on the SMC clusters NGC 361 and 419. This was followed by papers by Eggen and Sandage and by the writer on NGC 1783 in the LMC, and by Tifft (1962) on NGC 121 in the SMC. Of these four clusters only NGC 121 appeared really similar to a galactic globular cluster, the others displaying features not reproduced by any known cluster in the Galaxy. Further work was clearly needed to clarify the problems raised by these results, and a program for the systematic observation of the colour-magnitude diagrams of red clusters in the Clouds was accordingly begun here in September 1961. This contribution is a progress report on this program.

The observational material consists of blue and yellow plates taken on the 74inch telescope, calibrated by sequences observed with a photoelectric photometer on the 50-inch. In favourable conditions, that is, relative freedom from field stars, the sequences could be carried to $V=19 \cdot 0$, and, with some loss of accuracy, to $V=19 \cdot 5$. Generally, however, the program was not aimed at extremely faint stars or very high accuracy, but rather at collecting reasonably good results for as many clusters as possible. We describe here results on six clusters - Kron 3 and NGC 339 in the SMC, and NGC 1466, 2231, an anonymous companion to 2231, and NGC 2257 , all in the LMC. Because of limitations on space we can give colour-magnitude diagrams for only two of these, and in Figure 1 present instead a schematic designed to show the main features of all clusters observed to date.

We first discuss NGC 1466 and 2257, in which the Pretoria observers have discovered 46 and 27 variables respectively, mostly of short period. Their colourmagnitude diagrams are similar, both with well-defined horizontal branches, strongly concentrated towards the blue side, and equally well-marked giant branches, the termination points of which lie about $3.0 \mathrm{mag}(V)$ above the horizontal branches. In these respects NGC 1466 and 2257 resemble closely the metal-poor halo clusters, 